\title{
PKAIN: an artificial immune network for parameter optimization in pharmacokinetics
}

\author{
L. Liu ${ }^{1}$, C.-H. Lai ${ }^{2}$, S.-d. Zhou ${ }^{3}$, F. Xie ${ }^{3}$ \& H.-w. Lu ${ }^{3}$ \\ ${ }^{1}$ School of Information Technology, Jiangnan University, Wuxi, \\ Jiangsu, P. R. China \\ ${ }^{2}$ School of Computing and Mathematical Science, \\ University of Greenwich, London, UK \\ ${ }^{3}$ Suzhou University Affiliated Forth People's Hospital, \\ The Forth People's Hospital of Wuxi, Wuxi, Jiangsu, P. R. China
}

\begin{abstract}
The PKAIN algorithm is an artificial immune network, which has been designed to optimize parameters of linear pharmacokinetic models in our previous work. In this paper, the algorithm is modified to optimise parameters of nonlinear pharmacokinetic models. To evaluate parameters, the numerical inverse Laplace method is adopted to calculate drug concentrations of the dynamic system. The initial solutions of pharmacokinetic parameters are generated randomly by the PKAIN algorithm in a given solution space. Memory cells to be used in the search of global optimal parameters are generated. The optimal mechanism of the algorithm is based on artificial immune network principles and simplex mutation. In addition, a distributed version of the PKAIN algorithm is proposed to improve its efficiency.
\end{abstract}

Keywords: pharmacokinetic model, distributed computing, artificial immune network, numerical inverse Laplace, simplex.

\section{Introduction}

The artificial immune system [1] is a novel soft computing paradigm, which simulates powerful abilities of biological immune systems in mutual action to defend against pathogenic organisms. Studies on the comparison of optimization performances between the artificial immune system and other heuristic algorithms [2], such as simulated annealing algorithm, genetic algorithm, and 
evolution programming, have demonstrated that the artificial immune system has outstanding characteristics of population diversity and global convergence. Furthermore, the artificial immune network has been successfully used in multimodal function optimization [3], and dynamic environment optimization [4]. Various applications of the artificial immune network are currently being studied by various research groups.

In order to understand absorption, distribution, metabolization and elimination processes of a drug, a pharmacokinetic model is required to be built with suitable model parameters. Optimised parameters are usually determined through the use of observed drug concentrations after a period of time of applying the drug. Therefore the optimisation process is the most fundamental and important task in establishing a robust pharmacokinetic model. In our previous work, parameters of a linear compartment model are optimized [5]. First, the Laplace transform is applied to the linear compartment model. Analytical inverse Laplace transform is used to deduce the drug concentration function. The artificial immune network PKAIN was developed to optimise parameters of the concentration function. In contrast to Gauss-Newton and simplex methods, the PKAIN is capable of obtaining the global optimal solution and the process is insensitive to initial solutions.

In this paper, the PKAIN method is extended to optimise parameters of nonlinear pharmacokinetic models. Unlike linear compartment models, the concentration function of nonlinear pharmacokinetic model tends to be unavailable in its analytic form. Therefore the numerical inverse Laplace method is used to obtain discrete concentrations in a given temporal period for the given nonlinear model. In order to optimize parameter, the PKAIN is modified as follows. First, fitness calculation integrated with numerical inverse Laplace method is developed. Second, a distributed version of the PKAIN algorithm is proposed to improve its efficiency.

\section{The artificial immune network for parameter optimization of nonlinear pharmacokinetics}

In the PKAIN algorithm, a set of parameters describing a given pharmacokinetic model is encoded into a memory cell of the artificial immune network. The procedure of the PKAIN artificial immune network for parameter optimization of nonlinear pharmacokinetics is described as follows. Initially, the memory cells of the artificial immune network are randomly generated in the solution space. For each cell, clone selection and mutation are used to generate new cells. The fitness of each cell is calculated by evaluating the 'goodness' of the set of parameters and are encoded into them. In the network suppression process, similar cells are deleted to maintain a relatively smaller network scale. Certain proportional cells with lower fitness are updated dynamically and a new generation of the network is generated. The network is allowed to evolve until certain stopping criterion has been achieved. Finally, the memory cell with the highest fitness is decoded and the optimal set of parameters of the pharmacokinetic model is obtained. In the following subsections, the fitness 
calculation, clone selection and simplex mutation processes of the PKAIN are described.

\subsection{Fitness calculation}

Let $c=\left\{\theta_{1}, \theta_{2}, \cdots, \theta_{l}\right\}$ denotes a set of $l$ parameters describing a pharmacokinetic model. A candidate solution of the parameters is encoded into a memory cell. The fitness of a network cell is related to an objective function which is used to evaluate the goodness of the cell. In the context of pharmacokinetic model optimization, it is a measure of how well the drug concentration computed by using the set of parameters of the model would fit with the observed drug concentration. The fitness is measured as a numerical value ranging from 0 to 1 and the closer the fitness value is to 1 means that the better the set of parameters encoded in a memory cell is to fit the observation. Let $x_{i}, i=1,2, \ldots, n$ be the drug concentration observed at time $t_{i}$ and $X_{i}$ the calculated drug concentration using $c$ for the nonlinear pharmacokinetic model, the fitness of a memory cell $\eta(c)$, may be defined as follow:

$$
\eta(c)=1-\sum_{i=1}^{n} w_{i}\left(x_{i}-X_{i}\right)^{2} / \sum_{i=1}^{n}\left(x_{i}-\bar{x}\right),
$$

where $\bar{x}$ is the average observed drug concentration. $w_{i}$ is set to the value 1 , otherwise $1 / x_{i}$, or $1 / x_{i}{ }^{2}$ when $x_{i}$ exhibits a wide range of values.

Consider the nonlinear pharmacokinetic model of which the drug concentration $X_{i}=X\left(t_{i}\right)$ is required:

$$
\frac{d X}{d t}=A(X)+H(X)+f(t),
$$

where $A(X)$ is a linear function of $X, H(X)$ is a nonlinear function of $X$, and $f(t)$ is a known function of $t$. To obtain numerical solutions of the nonlinear pharmacokinetic model, the iterative coefficient-inverse Laplace method (ICIL), which was successful used in our previous work [6] for nonlinear Black-Scholes equations in financial computing, is adopted.

At each time step, a linearization of the nonlinear term $H(X)$ is obtained by computing $H(\widetilde{X})$ using the approximation $\widetilde{X}$, which is updated within an iterative update process in order for $\widetilde{X}$ to converge to $\widetilde{X}$. Each step of the iterative update process involves a numerical solution to the equation,

$$
\frac{d X}{d t}=A(X)+H(\widetilde{X})+f(t),
$$

where Laplace transformation may be applied easily. Let $X_{p}$ and $X_{p+1}$ be the numerical solutions of eq. (3) at $t=t_{p}$ and $t=t_{p+1}$ respectively. Also let $U(\lambda)$ and $F(\lambda)$ be the Laplace transforms of $X$ and $f(t)$ respectively, where $\lambda$ is the parameter introduced after taking the Laplace transform. Applying the Laplace transform to eq. (3), being defined in the time interval $t \in\left[t_{p}, t_{p+1}\right]$, in its differential form leads to

$$
U(\lambda)=\left[H(\widetilde{X}) / \lambda+F(\lambda)+X_{p}\right] /(\lambda-A),(\lambda-A \neq 0)
$$


The iterative update process to obtain the numerical solution $X_{p+1}$, using $X_{p}$ as the initial approximation to $\tilde{X}$ and assuming $m$ parametric functions of $U\left(\lambda_{j}\right), j=1,2, \ldots, m$, is described in the ICIL algorithm as below.

\section{FUNCTION to find $X_{p+1}$}

Initial approximation: $X_{p+1}^{(0)}=X_{p}$

//Iterative Inverse Laplace Method in $t \in\left[t_{p}, t_{p+1}\right]$

$\mathrm{k}=0$;

ITERATE

$$
\tilde{X}=X_{p+1}^{(k)} \text {; }
$$

Compute $U\left(\lambda_{j}\right)=\left[H(\tilde{X}) / \lambda_{j}+F\left(\lambda_{j}\right)+X_{p}\right] /\left(\lambda_{j}-A\right), j=1,2, \ldots, m$

$$
\text { where } \lambda_{j}=j \frac{\ln 2}{t_{p+1}-t_{p}}
$$

Find $X_{p+1}^{(k)} \approx \frac{\ln 2}{t_{p+1}-t_{p}} \sum_{j=1}^{m} w_{j} U\left(\lambda_{j}\right)$

$$
\text { where } \lambda_{j}=j \frac{\ln 2}{t_{p+1}-t_{p}} \text {; }
$$

$$
w_{j}=(-1)^{m / 2^{+j}} \sum_{k=(1+j) / 2}^{\min (j, m / 2)} \frac{k^{m / 2}(2 k) !}{(m / 2-k) ! k !(k-1) !(j-k) !(2 k-j) !} ;
$$

$$
k=k+1 \text {; }
$$

UNTIL $\left\|X_{p+1}^{(k)}-X_{p+1}^{(k-1)}\right\|<\varepsilon$

$X_{p+1}=X_{p+1}^{(k)}$;

\section{END-FUNCTION}

\subsection{Clonal selection and simplex mutation}

The clonal selection process of an artificial immune network is a computational implementation of the clonal selection principle in solving optimization problems, emphasizing multimodal and combinatorial optimization [7]. The process of clonal selection is composed of clone, mutation and selection steps. In the clone step, identical off springs of memory cells are generated. Then new cells are created through the mutation step. The PKAIN algorithm executes clone and fitness-based mutation steps as below.

$$
\begin{array}{r}
c_{\text {new }}=c+\alpha N(0,1) \\
\alpha=(1 / \beta) \exp \left(-\eta^{*}(c)\right)
\end{array}
$$

where $c_{\text {new }}$ is a new memory cell mutated from the cell $c, N(0,1)$ is a Gaussian random variable of zero mean with deviation $\sigma=1$. The mutation factor $\alpha$ is inversely proportional to the clone constant $\beta . \eta^{*}(c)$ is the normalized fitness of 
the cell $c$. In this case, cells with low fitness mutate heavily so that they have many chances to be better than their parents to improve the diversity of the population. On the contrary, cells with higher fitness mutate relatively little to reserve their priority.

In order to improve local optimal capability, the simplex mutation is designed. In the classical simplex method, a simplex is constructed by its $L+1$ vertices in the $L$ dimensional Euclidean space. Then, through a sequence of elementary geometric transformations (reflection, contraction, expansion and shrinking), the initial simplex moves, expands and contracts. In such a way that it adapts itself to the function landscape and finally surrounds the optimum [8]. Since the simplex method is an excellent method for local optimization, it has been used with other searching techniques in a hybrid fashion. Yen et al. [9] developed a variant of the concurrent simplex method which begins with $L+\Omega$ points, where $\Omega>1$, instead of $L+1$ points as in the classical simplex method.

In this paper, a partition-based concurrent simplex mutation is examined. The new cells $c_{\text {new }}$ generated by the clone and mutation steps of the clonal selection process for a given memory cell $c$ are considered as a natural partition group. The number of simplex mutated cells is denoted as $N_{c}, N_{c}>L+1$. After executing the concurrent simplex method to obtain $c_{\text {new }}$, there are $N_{c}-L$ number of new cells that have been updated. The partition-based concurrent simplex mutation is described as the following steps.

(1) Order

Order the cells of $c_{\text {new }}$ to satisfy

$\eta\left(c_{1}\right) \geq \ldots \geq \eta\left(c_{L}\right) \geq \ldots \geq \eta\left(c_{L+i}\right) \geq \ldots \geq \eta\left(c_{N_{c}}\right), i=1,2, \ldots, N_{c}-L$.

Calculate the centroid of $L$ number of cells with higher fitness values, $\bar{c}=\left(c_{1}+c_{2}+\ldots+c_{L}\right) / L$.

(2) Reflection

For each $c_{L+i}$ cell, compute the reflection point $c^{r}$ by using

$$
c^{r}=\bar{c}+\rho\left(\bar{c}-c_{L+i}\right)
$$

Calculate the fitness $\eta^{r}=\eta\left(c^{r}\right)$. If $\eta\left(c_{L}\right)<\eta^{r}<\eta\left(c_{1}\right)$, accept $c_{L+i}=c^{r}$ and terminate the operation.

(3) Expansion

If $\eta^{r} \geq \eta\left(c_{1}\right)$, expand the point $c^{r}$ to $c^{e}$ by using

$$
c^{e}=\bar{c}+\rho \chi\left(\bar{c}-c_{L+i}\right)
$$

Calculate the fitness $\eta^{e}=\eta\left(c^{e}\right)$. If $\eta^{e}>\eta^{r}$, accept $c_{L+i}=c^{e}$ and terminate the operation; otherwise, accept $c_{L+i}=c^{r}$ and terminate the operation. 
(4) Outside Contraction

If $\eta\left(c_{L+i}\right)<\eta^{r}<\eta\left(c_{L}\right)$, compute the outside contraction point $c^{o}$ as

$$
c^{o}=\bar{c}+\rho \gamma\left(\bar{c}-c_{L+i}\right)
$$

Calculate the fitness $\eta^{o}=\eta\left(c^{o}\right)$. If $\eta^{o}>\eta^{r}$, accept $c_{L+i}=c^{o}$ and terminate the operation.

(5) Inside Contraction

If $\eta^{r}<\eta\left(c_{L}\right)$ and $\eta\left(c_{L+i}\right) \geq \eta^{r}$, compute an inside contraction point $c^{i}$ as

$$
c^{i}=\bar{c}-\gamma\left(\bar{c}-c_{L+i}\right)
$$

Calculate the fitness $\eta^{i}=\eta\left(c^{i}\right)$. If $\eta^{i}>\eta\left(c_{L+i}\right)$, accept $c_{L+i}=c^{i}$ and terminate the operation.

There are coefficients of reflection $(\rho)$, expansion $(\chi)$, and contraction $(\gamma)$. The usual choices of these coefficients are $\rho=1, \chi=2, \gamma=0.5$. The shrinking operator of the classical simplex method is replaced with the mutation step of the clonal selection process.

\section{A distributed PKAIN method}

In order to obtain numerical solution of eq. (3) accurately by means of a temporal integration method, temporal intervals $\left[t_{p}, t_{p+1}\right]$ should be small. Unfortunately, concurrent computation of all time steps in a temporal integration method is impossible. It seems that to achieve a distributed algorithm to yield a de-coupling of the original problem is almost impossible. However in the ICIL algorithm, the numerical solutions $X_{p}, p=1, \ldots, N$ may be computed concurrently and, thus, the total computational time of becomes significantly reduced.

In this paper, we examine a two-level temporal decomposition method which stems from our previous work of concurrency in time domain computation [10, 11]. Assuming the $N$ temporal steps of serial calculation are equally divided into $N_{\text {coarse }}$ parts, each represents a coarse temporal interval $\Delta T$. In the secondary temporal decomposition, each $\Delta T$ is divided into $N_{\text {fine }}$ parts of finer temporal intervals $\Delta t, \Delta T=N_{\text {fine }} \cdot \Delta t$. First, a number of numerical solutions of the nonlinear equation, using the concept of the Laplace transform and its iterative inverse, are obtained on a coarser temporal mesh, $t \in\left[T_{p-1}, T_{p}\right]$, $\mathrm{p} \in\left\{1, \ldots, \mathrm{N}_{\text {coarse }}\right\}$. Second, each coarse temporal mesh $t \in\left[T_{p-1}, T_{p}\right]$ can be decomposed into several finer temporal meshes $t \in\left[T_{p-1}, T_{p-1}+t_{i}\right]$, $\mathrm{i} \in\left\{1, \ldots, N_{\text {fine }}-1\right\}$. The numerical solutions on the coarser temporal mesh obtained in the former step are used as initial conditions on the finer temporal mesh. Solutions defined on a finer temporal mesh for each of the coarse temporal mesh are now being obtained concurrently using a temporal integration method. This linearization leads to a distributed version of the fitness calculation process 
for the PKAIN. Suppose the fitness calculation can be distributed into $p$ processors, the pseudocode of the fitness calculation is described as below.

FUNCTION Distributed fitness calculation

Decode the network cell $c$ into parameters.

FOR $\mathrm{p}=1$ TO $\mathrm{N}_{\text {coarse }}$, do coarser temporal mesh on $t \in\left[T_{p-1}, T_{p}\right]$

Find $X_{p}$ from $X_{p-1}$;

END-FOR

Distribute $\mathrm{p}=0$ TO $\mathrm{N}_{\text {coarse }}-1, X_{p}$ to processors,

For $\mathrm{i}=1 T \mathrm{o} \mathrm{N}_{\text {fine }}-1$, do finer temporal mesh on $t \in\left[T_{p}, T_{p}+t_{i}\right]$

Find $X_{p+i}$ from $X_{p+(i-1)}$;

END-Distribute and receive $X_{p+i}$.

$$
\eta=1-\sum_{i=1}^{n} w_{i}\left(x_{i}-X_{i}\right)^{2} / \sum_{i=1}^{n}\left(x_{i}-\bar{x}\right)
$$

\section{END-FUNCTION}

\section{Experiments and discussion}

A typical nonlinear pharmacokinetic model for drug concentration is often described by Michaelis-Menten equation. In this section, the PKAIN algorithm is used to optimize Michaelis-Menten pharmacokinetic parameters of the bolus intravenous examples 1 and 2 as described in [12]. Optimal parameters are compared to the solutions given by the accurate linear regression (ALR), improved Hanes-Woolf method (HW), and combined Runge-Kutta method (RKPS) in the literature.

The equation of drug concentration described by Michaelis-Menten equation consists of two parameters is given as follow.

$$
\frac{d X}{d t}=-\frac{V_{m} X}{K_{m}+X}
$$

Table 1: $\quad$ Comparison of optimal parameters for example 1.

\begin{tabular}{l|lll}
\hline Method & \multicolumn{1}{|l}{$K_{m}$} & $V_{m}$ & $R$ \\
\hline PKAIN & 10.907 & 4.016 & 0.042690 \\
HW & 10.5135 & 3.9615 & 2.260105 \\
ALR & 9.3936 & 3.9573 & 0.293910 \\
RK-PS & 9.9804 & 3.9994 & 0.111829 \\
\hline
\end{tabular}

In other words, candidate solutions of $c=\left\{V_{m}, K_{m}\right\}$ are encoded into memory cells. Optimal parameters for example 1 obtained by PKAIN, ALR, HW, and RK-PS methods are shown in Table 1. Optimal parameters for example 2 are shown in Table 2. In order to compare these parameters, the relative residual 
$R=\sum\left(\left(x_{i}-X_{i}\right)^{2} / x_{i}\right)$ is calculated. The smaller the weighted residual is, the better the parameters are. The results demonstrated that the PKAIN method outperforms HW, ALR, and PK-PS algorithms in parameter optimization of nonlinear pharmacokinetic models.

Table 2: $\quad$ Comparison of optimal parameters for example 2.

\begin{tabular}{l|lll}
\hline Method & $K_{m}$ & $V_{m}$ & $R$ \\
\hline PKAIN & 33.2223 & 13.4414 & 1.5718282 \\
HW & 28.0806 & 11.2978 & 9.292684 \\
ALR & 27.5851 & 11.2828 & 8.509200 \\
RK-PS & 29.5126 & 13.0842 & 1.817844 \\
\hline
\end{tabular}

\section{Conclusions}

In this paper, the artificial immune network PKAIN is designed to optimize nonlinear pharmacokinetic parameters. The method to obtain numerical solutions of nonlinear system is integrated into its fitness calculation process. Experimental results obtained by the PKAIN algorithm are better than those obtained by HW, ALR, and PK-PS methods. Together with our previous work, the PKAIN algorithm is capable of obtaining optimal parameters for both linear and nonlinear pharmacokinetics. In addition, the two-level temporal decomposition method is used to parallelize the nonlinear pharmacokinetic model using an iterative inverse Laplace transformation. The efficiency of the PKAIN algorithm can be greatly improved when it is implemented in a distributed environment due to the fact that there is no data dependence of the fine temporal mesh computation.

\section{Acknowledgement}

The authors acknowledge support from innovation team project JNIRT0702 of Jiangnan University.

\section{References}

[1] de Castro, L.N., Timmis, J., Artificial immune systems as a novel soft computing paradigm. Soft Computing, 7(8), pp.526-544, 2003.

[2] Xie, K.G., Zeng, X.H., Li, C.Y., et al., Comparative analysis between immune algorithm and other random searching algorithms. Journal of Chongqing University, 26(11), pp.43-47, 2003, (in Chinese).

[3] de Castro, L.N, Timmis, J., An artificial immune network for multimodal function optimization. Proc. of IEEE Congress on Evolutionary Computation, IEEEE Service Center, Honolulu, USA, pp.674-699, 2004.

[4] de Franca, F.O., Von Zuben, F.J., de Castro, L.N., An artificial immune network for multimodal function optimization on dynamic environments. 
Proc. of the GECCO conf, ACM Press, Washington DC, pp. 289-296, 2005.

[5] Liu, L., Zhou, S.D., Lu, H.W., et al., Parameter optimization of pharmacokinetics based on artificial immune network. Applied Mathematics And Mechanics-English Edition, 29(4), pp.549-559, 2008.

[6] Lai, C.H., Parrott, A.K., Rout, S., A Distributed Algorithm for European Options with Nonlinear Volatility. Computers and Mathematics with Applications, 49, pp.885-894, 2005.

[7] de Castro, L.N., Von Zuben, F.J., Learning and optimization using the clonal selection principle. IEEE Trans on Evol Comp, 6(3), pp.239-251, 2002.

[8] Jeffrey, C.L., James, A.R., Margaret, H.W., et al., Convergence properties of the Nelder-Mead simplex method in low dimensions. Society for Industrial and Applied Mathematics, 9(1), pp.112-147, 1998.

[9] Yen, J., Liao, J., Randolph, D., Lee, B., A Hybrid approach to modelling metabolic systems using a genetic algorithms and the simplex method. IEEE Transactions on Systems; Man; and Cybernetics; 28(2), pp.173-191, 1998.

[10] Lai, C.H., On transformation methods and concurrency in time domain computation. Proc. of the DCABES 2007, pp. 5-6, 2007.

[11] Lai, C.H., Numerical solutions of certain nonlinear models in European options on a distributed computing environment. Nonlinear Models in Mathematical Finance: New Research Trends in Option Pricing, ed. Matthias Ehrhardt, Nova Science Publisher, pp.283-298, 2008.

[12] Su, Y.F., Optimizing method of Michaelis-Menten pharmacokinetic parameters of bolus intravenous administration. Chin J Clin Pharmacol Ther 10(10), pp.1198-1200, 2005, (in Chinese). 